\title{
22
}

\section{Automatic Updates of Interactive Information Visualization User Interfaces through Database Triggers}

\author{
Martin Leissler, Matthias Hemmje, Erich Neuhold \\ GMD - German National Research Center for Information Technology \\ IPSI - Integrated Publication and Information Systems Institute
}

Dolivostr. 15, 64293 Darmstadt, Germany

[leissler, hemmje, neuhold]@darmstadt.gmd.de

\begin{abstract}
In client-server based interactive information visualization, user interfaces data sets of many types are made accessible by a server. On the server side, these data sets can, e.g., be stored in a file system. As soon as a user requests the data set, it will be transmitted from the server to the requesting client. A different approach is to dynamically construct a data set at request time on the server side for visualization on the client side. Another approach for database driven information visualization is to request actual data out of a running information visualization application on the client side. Both visualization methods have in common that changes in the underlying data cannot be automatically reflected in changes of the client's information visualization state and context. A solution for this problem is the use of database triggers which today are often implemented in existing database management systems (DBMS). Database triggers are a mechanism used to define a set of operations which are to be executed by the DBMS as soon as a given part of the data in the database changes. Using triggers to update an information visualization client has the advantage of eliminating unnecessary database requests. Moreover, the delay in updating the information visualization on the client side is only dependent on the time it takes to send a message from the server to the client. This paper describes a conceptual model for such a trigger mechanism and evaluates some upcoming problems. Furthermore, the paper describes a prototypical proof-of-concept implementation based on commonly used technologies and systems.
\end{abstract}


Keywords: Information Visualization, Database Trigger, Active Databases, User Interfaces

\section{INTRODUCTION AND MOTIVATION}

In client-server-based interactive information visualization user interfaces (see, e.g., [LHN99], [MLHN99], [RLHA98], [MSSNHPL98]), data sets of many types are made accessible by a server. Users request such data sets and client user interfaces visualize them applying a given information visualization mapping. On the server side, these data sets can, e.g., be stored in a file system. As soon as a user requests the data set, it will be transmitted from the server to the requesting client. A different approach is to dynamically construct a data set at request time on the server side for visualization on the client side. This approach is often used in databasedriven information visualization applications. Upon receipt of users' requests a program is executed on the server which retrieves the corresponding data from a database and generates a data set from it (and possibly some additional meta data) .

A different approach for database-driven information visualization is to request actual data out of a running information visualization application on the client side. An information visualization application can therefore request data at an arbitrary point in time and change its information visualization state and informational context based on the incoming result data.

Both visualization methods have in common that changes in the underlying data cannot be automatically reflected in changes of the client's information visualization state and informational context. With dynamic construction at request time, it would be necessary to retrieve a completely new data set from the server every time the database content changes. With runtime access, one would need to trigger new requests from the visualization client.

To overcome these limitations, it would be possible in general to let the client query the database for possible changes in regular intervals. This approach has the obvious drawback that a potentially large number of database queries would be executed without the underlying data ever having changed. This would in turn result in unnecessary system and network load. Furthermore the information visualization client would not be updated immediately but would have to wait until the next data retrieval.

A better solution for this problem is the use of database triggers which, today, are often implemented in existing database management systems (DBMS) [WC96a]. Database triggers are a mechanism used to define a set of operations which are to be executed by the DBMS as soon as a given part of 
the data in the database changes. Using triggers to update an information visualization client has the advantage that no unnecessary database requests have to be made and that the delay in updating the information visualization on the client side is only dependent on the time it takes to send a message from the server to the client. The use of triggers for the notification of VRML scenes was already postulated in [VDBGW97].

This paper describes a conceptual model for such a trigger mechanism and evaluates some upcoming problems. Furthermore, it will describe a prototypical proof-of-concept implementation based on commonly used technologies and systems.

The reader is assumed to have some basic knowledge in the area of DBMSs as can be gained from [EN94]. Furthermore, it is assumed that a basic understanding of VRML (see [ISO97a]), the external authoring interface (short EAI [Mar97]), and the TCP network protocol [RFC793] is given.

\section{ACTIVE DATABASE MANAGEMENT SYSTEMS}

Conventional DBMS are passive, which means they will only perform operations on data when explicitly queried by an application. No other operations are performed. Active DBMSs (ADBMS), however, are able to perform operations which are not explicitly defined by an application. A typical use for an ADBMS are operations which are performed as a direct reaction to modification operations (in SQL: Insert, Delete and Update) on the underlying data. (See descriptions of research prototypes in [WC96b]).

The active behaviour of an ADBMS can be generally described by a so called event-condition-action rule (ECA rule). If an event of an ECA rule occurs, the condition is tested and the action will be performed if the condition evaluates true.

Events can, for example, be changes on data or data queries but also be defined by some absolute point in time (e.g. 8/1/1999, 8:00 PM). Other events defined over time are described in [DBC96]. Furthermore, events can be defined by application programs. This could be achieved by registering an event under a given name. If the event occurs the application program calls the ADBMS referring to the given name.

All events described so far are simple events. However, events can also be composite events, which means they are defined on the basis of other events. A composite event can e.g. be defined by two simple events which have to occur sequentially. A system which supports composite events is for example HiPAC [DBC96]. 
The condition in an ECA rule is based on the internal state of the database, the event-related data, and other information accessible by the DBMS. Event-related data can be the new value and the old value of the changed data, e.g., in a data modification operation.

In [WC96c] the possible actions of an ECA rule are classified as

- Data modification operations

- Data retrieval operations

- Other database commands

- Application procedures.

In this context the case of application procedures shall be examined: An action can be defined by a call to a procedure defined in a programming language such as $\mathrm{C}$. Therefore it is possible to implement a notification of systems external to the database system if the procedure sends data to the external system.

\section{COUPLING MODES}

In the following, it is assumed that an event always occurs inside of a transaction (this would not apply to time-based events) which is the only relevant case in the context of this paper. Within the execution of an ECA rule there are different possibilities for the evaluation of the condition and the execution of the action relative to the transaction which triggered the event of the ECA rule (called triggering transaction). These possibilities are called coupling modes. In this context we will shortly describe the coupling modes defined in the HiPAC project [DBC96] and two other coupling modes from [BBKZ93].

In particular the coupling modes are: immediate, deferred, detached, parallel causally dependent, sequential causally dependent, and exclusive causally dependent. These modes can describe the evaluation of the condition in relation to the causal transaction as well as the execution of the action relative to the transaction in which the condition is evaluated. For reasons of simplicity only the evaluation of the condition is considered.

With immediate coupling the condition is evaluated immediately after the occurrence of the event inside the triggering transaction. The normal execution of the triggering transaction is stopped in the meantime. With deferred coupling the condition is evaluated at transaction end (but before the commit) inside of the triggering transaction. With decoupled coupling a new transaction is started for the evaluation of the condition which runs in parallel and independent of the triggering transaction. Parallel causally dependent is the same as detached except that the new transaction can only terminate regularly if the triggering transaction is terminated regularly. With 
sequential causally dependent a new transaction is started as in the two cases before. The execution start time, however, lies after the commit of the triggering transaction. That means, the new transaction is only started if the triggering transaction has committed. With exclusive causally dependent a new transaction is started for the evaluation of the condition which only reaches its commit-point if the triggering transaction is aborted. If the new transaction has already been started at the commit-point of the triggering transaction, it will be reset.

In HiPAC, all coupling modes can either be assigned to a condition or an action of an ECA rule. In other ADBMSs (e.g. Ode [GJ96]) the coupling modes cannot be explicitly set. However, it is possible to achieve an equivalent execution of ECA rules by using composite events [GJ96].

For broader discussions on ADBMS, it is recommended to read [WC96b, Day95, DGG95, Buc94].

\section{TRIGGERS IN SQL3}

This section shortly outlines how triggers are represented as described in a draft version of the SQL3 standard [ISO97b]. The representation is based on an interpretation of the draft standard by the author.

The general SQL3 syntax for a trigger is:

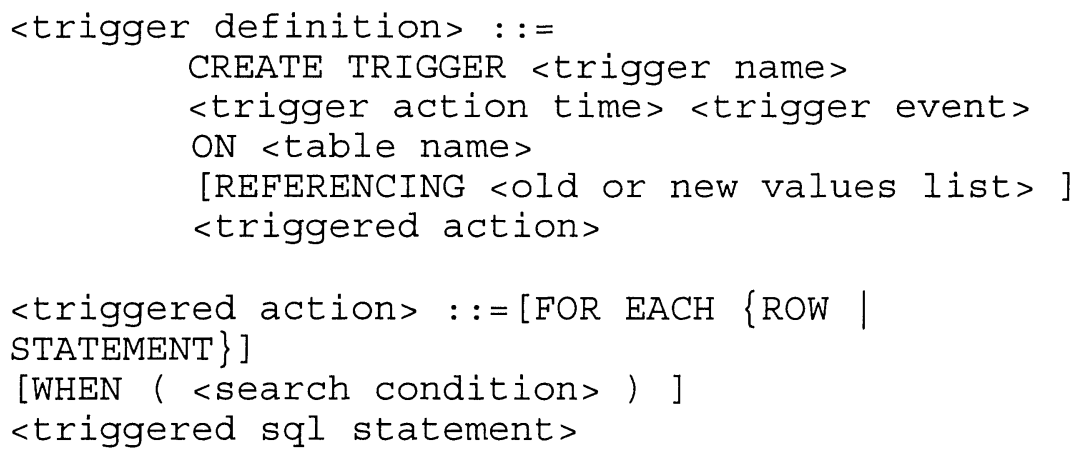

With the CREATE TRIGGER statement a new trigger with the name $<$ trigger name $>$ is defined in a database. <trigger event $>$ and $<$ table name $>$ define which operation has to be performed on which table to release the trigger. <search condition> defines a condition which has to be true for $<$ triggered sql statement $>$ to be executed. FOR EACH ROW defines that the trigger is executed for each row and FOR EACH STATEMENT defines that the trigger is only executed once for the whole causal statement. With $<$ trigger action time> set to BEFORE (AFTER) the trigger is executed before (after) the change in the table becomes permanent. With the 
REFERENCING keyword, names can be defined for old and new table values which can be referenced in the <triggered action>.

SQL3 triggers can be seen as ECA rules. The event is defined by the modifier operation INSERT, DELETE or UPDATE. <search condition> is equivalent to the condition and <triggered sql statement $>$ is equivalent to the action. For this paper the coupling between event condition and action is relevant. The coupling mode between the triggering event and the evaluation of the condition is immediate. The coupling mode between the evaluation of the condition and the execution of the action is also immediate. In the next section, we will see an obvious drawback of using this coupling mode for notifying external systems with the help of database triggers.

\section{ACTIVE DBMS AND EXTERNAL SYSTEMS}

In [BBKZ93] the coupling mode sequential causally dependent has been introduced (described in Section 3) to be able to handle systems external to the database in which actions cannot be undone (which is characteristic for database driven client/server information visualization scenarios). Given that the action of an ECA rule has executed an operation on some external system in immediate mode the operation on the external system has to be reset if the triggering transaction is reset because the underlying event has effectively never occurred. However, if the external operation cannot be undone the operation has been executed even though the triggering transaction has been reset. An example for an external operation that cannot be undone is described in [BBKZ93] with the opening of a valve through which a fluid runs. This problem does not only occur with immediate coupling mode, but generally whenever an action with irreversible consequences is executed on an external system before the triggering transaction commits (that means also with the modes deferred and parallel causally dependent).

With coupling mode set to sequential causally dependent the new transaction is started after the transaction which generated the new transaction has committed. Therefore it is ensured that the transaction from which the new transaction was started will never be reset after the new transaction has started. If, e.g., an action is executed with sequential causally dependent and the coupling for the condition is immediate and this action leads to an operation on an external system the external operation surely will not have to be reset.

In [HCD98] the described problem (of notifying an external system before the triggering transaction has committed) is called dirty dependent operation problem (short DDO problem). Furthermore, [HCD98] describes a 
software architecture in which application programs can register at the database for a certain event. As soon as the event occurs, the registered application is called. The DDO problem is overcome by delaying the notification of the application program about an occurring event until after the triggering transaction has committed.

\section{COUPLING OF TRIGGERS WITH INFORMATION VISUALIZATION APPLICATIONS}

\subsection{Requirements}

The basic problem has already been mentioned in the introduction and will be discussed here in more detail. A data set requested from a server is visualized across several client applications via some visualization component (e.g. a browser plug-in). The set visualizes information carried by data which is permanently kept in a database. The data is either read directly from the clients or the server reads from the database upon receipt of a request and then generates a file for an information visualization based on the received data. Figure 1 outlines this scenario with both variants for database driven information visualization.

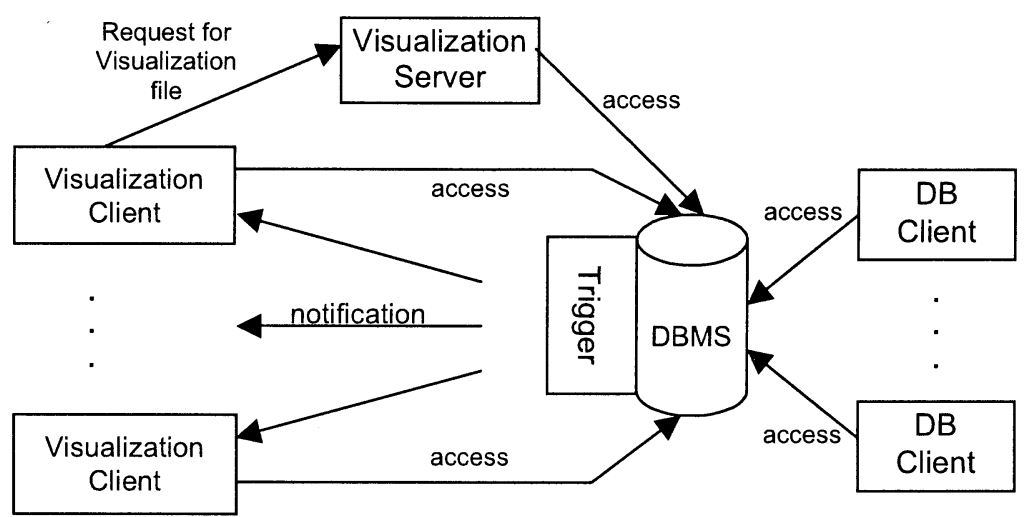

Figure 1: Environment of a system for the notification of information visualization clients through database triggers

Besides the visualization clients there are other clients which access the DBMS. Our goal is to have the database triggers notify the visualization clients about changes in the database in order to update the actual information visualization on the client side. This should also work with data 
modifications caused by existing software systems which have not yet been used in such a scenario.

The following requirements have been considered in the upcoming development of the architectural model:

- The system should not depend on specific features of the visualization clients

- The system should not depend on specific features of the underlying database system.

The first requirement (not to use client application specific features) is to ensure a broad usability across different client types. The second requirement is to make sure that the developed solution is easy to adapt to different DBMS systems.

\subsection{Trigger and External Systems}

In this section, we will examine how database triggers can be used to notify external systems about modifications in a database. It is assumed that the actions triggered by a notification of an external system cannot be reversed. This is in particular the case with information visualization applications. Information visualization applications typically wait for events either triggered by direct user interaction or from outside sources. As soon as the application handles a specific event, it modifies its visualization state which, in turn, results directly in a change of the screen shown to the user. Because the user perceives such changes immediately, they cannot be reversed afterward.

The results of this section should be applicable to SQL3, since SQL3 triggers are executed immediately within the triggering transaction (see Section 4). It is furthermore assumed that it is possible to notify an external system from the action part of a trigger in a given DBMS. We will explain later in detail how this is achieved in our prototypical implementation.

For now, there is the following problem: Since an action is executed within the triggering transaction, it is possible that a notification of the external system takes place although the transaction is reset afterwards. Because effectively no modification has occurred in the database there should be no notification of the external system, save we are interested in the information that a modification attempt has taken place (Note: This case is not considered further in this paper). This problem was already described generally for active databases above. Therefore it does not make sense to notify an external system about database modifications in the action of a trigger and consequently update a visualization on a user display because the 
modification has possibly never taken place. This problem can be tackled through at least two approaches:

- If the external system is interested in updating data from a database which is kept locally, an additional software component can be inserted between the DBMS and the external system which is notified by the action part of the trigger. The component interprets the notification to the effect that the data in the database which is mirrored locally has possibly changed. The relevant data is then read anew by a database query and sent to the external system.

- An additional software component is introduced which uses a database query to test upon notification by a trigger if the triggering transaction has terminated regularly. Only if the test is positive, the external system will be notified.

Both variants will be examined in more detail. It is furthermore assumed that applications which modify the database will reset the transaction via ROLLBACK if the modification is erroneous.

\subsubsection{Updating Data on Possible Changes}

Figure 2 is displays the general architecture of a system which queries a database (2) upon notification by a trigger (1). The results of the query (3) are then sent to the external system (4).

(1) trigger event

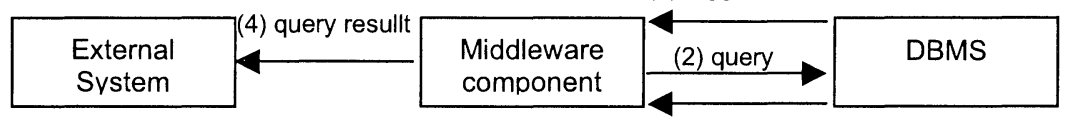

(3) query result

Figure 2: Architecture of a system in which data on an external system is updated as a reaction to a trigger event in a database.

The query assumes a newly introduced middleware component. An example for an external system could be an application which shows a stock index. The middleware component assures that the value shown on the screen always matches the actual value in the database.

If the DBMS keeps its write locks until the end of the transaction, the transaction which performs the query as a result of the notification by the trigger safely reads the modified values after the triggering transaction has ended because it has to wait until the triggering transaction releases its write locks. If the triggering transaction terminates regularly, the modified data is read. If the triggering transaction resets, the old unmodified data values are 
read again. But this is no problem since, in this case, the old values are still up to date. Only the read process costs some time.

In both cases only persistently stored data is read from the database. The problem that data which has not yet been stored permanently in the database is transmitted to an external system does not exist any more.

It is possible that the data is modified by another transaction before it is read by the middleware component. In this case the data modified by this other transaction is read immediately. The notification through this other transaction then leads to the same data being read again which is equivalent to the case of the rolled back transaction. In the worst case, we have an unnecessary database access.

\subsubsection{Checking if the Triggering Transaction has Terminated regularly}

The general architecture of a system which checks if the triggering transaction has terminated regularly after the action part of the trigger has been executed is displayed in Figure 3.

(1) trigger event

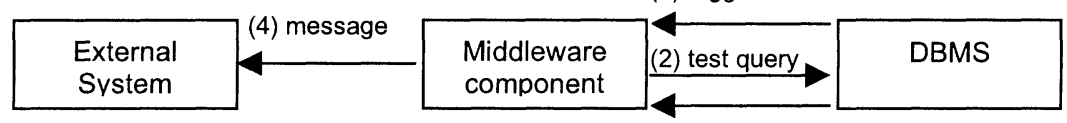

(3) query result

Figure 3: Architecture of a system for the notification of an external system through a database trigger which checks if the triggering transaction terminates regularly.

As in the previous case it involves a middleware component between the DBMS and the external system. If the action part of a trigger notifies the middleware component (1), the component performs a test query on the database (2) to test whether the triggering transaction has terminated regularly. If the test is positive (3) the external system is notified (4). How this can be done in detail is outlined by the following example of an UPDATE trigger.

It is assumed that the following tables are stored in a database:

$$
\begin{aligned}
& \text { Triggering }\left(\mathrm{N}_{1} \mathrm{~T}_{1}, \ldots, \mathrm{N}_{\mathrm{n}} \mathrm{T}_{\mathrm{n}}\right) \\
& \text { Counter }(\mathrm{c} \text { INTEGER) }
\end{aligned}
$$

The table Triggering is a table on which a trigger is to be defined. Its columns have the names $N_{1}, \ldots, N_{n}$ and the data types $T_{1}, \ldots, T_{n}$. The table Counter is an auxiliary table which holds only one row containing a single value. A trigger shall be defined on the table Triggering as follows: 


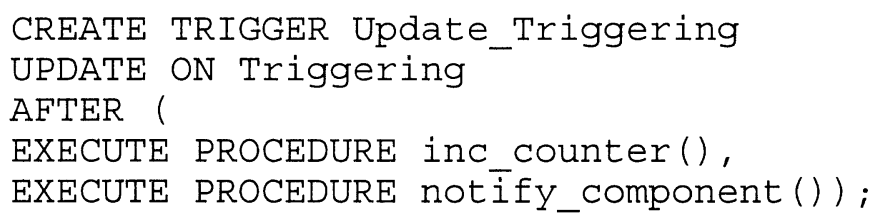

The procedure inc_counter() increments the value stored in the Counter table by one. If the maximum integer value given by the system is reached, it proceeds with the smallest possible value. The procedure notify_component() notifies the middleware component. Again we assume that the DBMS keeps the write locks until the end of a transaction.

The middleware component has an internal counter which is initialized to 0 in the beginning. The value in the table Counter is also set to 0 by the middleware component. Now if rows in the table Triggering are modified the action part of the trigger is executed. The value of the table Counter is incremented by 1 and the middleware component is notified. In the middleware component the table counter is read and compared to the internal counter value. If the value of the table Counter is not equal to the value of the internal counter, the external system is notified and the internal counter is incremented by 1 . Of course, it is assumed that both counters have the same range for their values and that the internal counter also proceeds with the minimal value if the maximum value is exceeded. If, however, the internal counter is equal to the value of the table Counter, the external system is not notified. If the middleware component receives notifications which cannot be processed immediately, they are buffered.

With this approach it is assured that a notification is only performed after the triggering transaction has committed and therefore only if the data modification that caused the trigger event has been stored permanently in the database. The reason for this can be explained as follows:

The triggering transaction occupies the value in the table Counter with a write lock until the end of the transaction because the action part of the trigger increments the value in the table Counter. The transaction with which the middleware component reads the table Counter has to wait until the triggering transaction has ended. At the point of commit, the incremented value remains in the database table, which means its value is unequal to the value of the components internal counter. The externals system is notified as a result. If, however, the triggering transaction is reset, the increment of the value in the table Counter is undone and the external system is not notified because the internal counter value is equal to the table Counter value. It is also possible that another transaction releases the trigger after the triggering transaction but before the value of the table Counter is read by the middleware component. Because the difference between both counters is equivalent to the number of triggering transactions which have terminated 
regularly but have not yet led to a notification of the external system, the external system is not notified too often. It is assumed that the value in the table Counter never hurries too far ahead of the internal counter such that both counters become equal again.

With this approach, it should even be possible to send the data which is made available by the trigger to the external system. If the database trigger is generated with the FOR EACH ROW keywords, it is possible to access the old and new data row values within the triggers action part. If these values are temporarily stored in a table or sent with the notification procedure a transfer to the external system should be possible. This approach is not further considered in this paper.

\subsection{Distribution of Messages}

In the previous section, it was implicitly assumed that there is a fixed external system which is notified if the action part of a trigger is executed. However, a client-server based system for the notification of information visualization clients has to notify every client that visually displays a local set of data. It is generally not known in advance which clients will visualize a given portion of data because the data can be requested from the server by an arbitrary client machine.

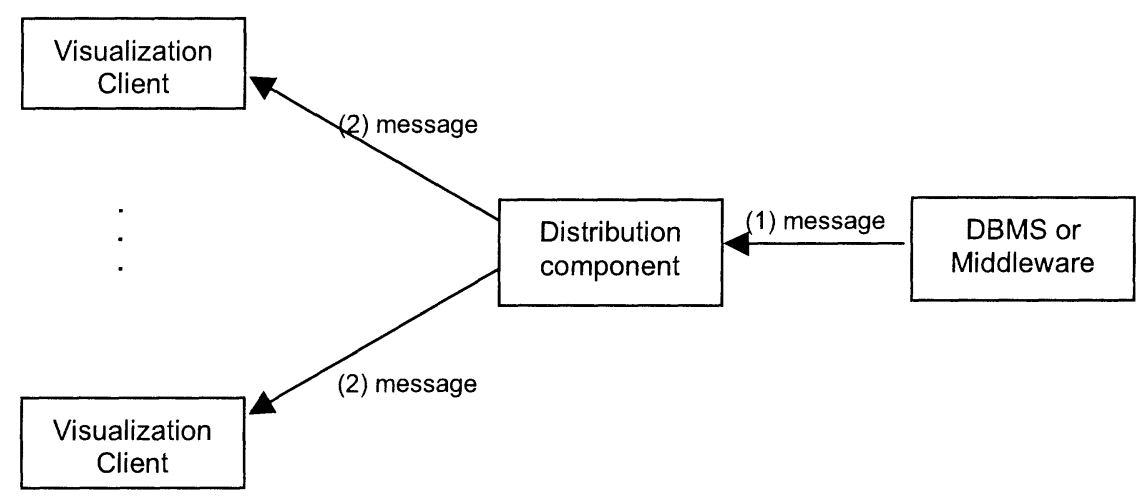

Figure 4: Distribution of messages across clients

For the distribution of messages to the client there could be a special system component. In Figure 4 the general architecture is displayed. If the distribution component receives a message (1), it is sent through to the clients (2).

At this time it is not described in detail how the distribution component knows which clients to notify and which protocol to use. 


\subsection{Discussion of Architectures}

This section describes possible architectures of a system for the notification of information visualization clients through database triggers. The different possibilities result from a combination of the general architectures depicted in Figure 2 and Figure 3 with the partial architecture for the distribution of messages as depicted in Figure 4. Furthermore, the possibility of accessing a database directly from an information visualization client is considered.

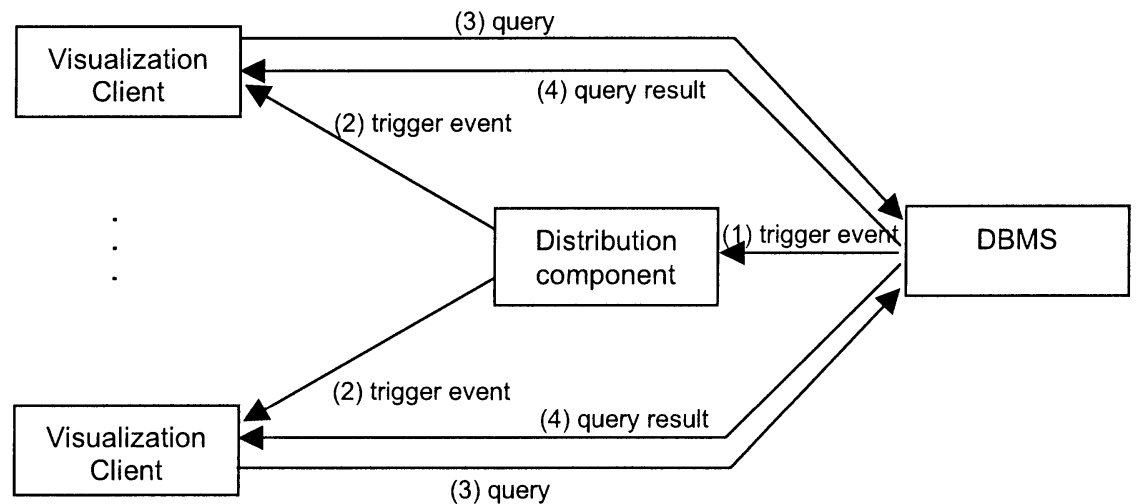

Figure 5: Architecture of a system in which information visualization clients are updated as a result of a trigger being executed.

Figure 5 and Figure 6 display architectures of systems which are based on the architecture of Figure 2. In the architecture shown in Figure 5, the functionality of the middleware component is fully performed by the information visualization clients. If the action part of a trigger notifies the clients (2) via the distribution component (1) that a data modification has possibly taken place, the clients perform database queries (3) and receive the results (4). Dependent on the query results, the clients update their visual state. Thereby it is absolutely possible that different clients send different queries to the database system. In a scenario in which the same information visualization application runs across several clients, this can, e.g., happen if through user interaction with the application, different parts of the overall database are to be visualized on different clients and therefore different queries have to be run against the database.

In Figure 6 though it is assumed that all clients visualize the same content partition of the database. The middleware component works as described before in 6.3. The distribution component performs the distribution of the data to the clients. The advantage of this architecture over the previous architecture is that only one database query has to be performed to update the information visualization clients, which clearly reduces the load for the DBMS. If, in the previous architecture (of Figure 5) all clients performed the 
same database query against the DBMS, the database would execute the same query multiple times which would also generate unnecessary workload.

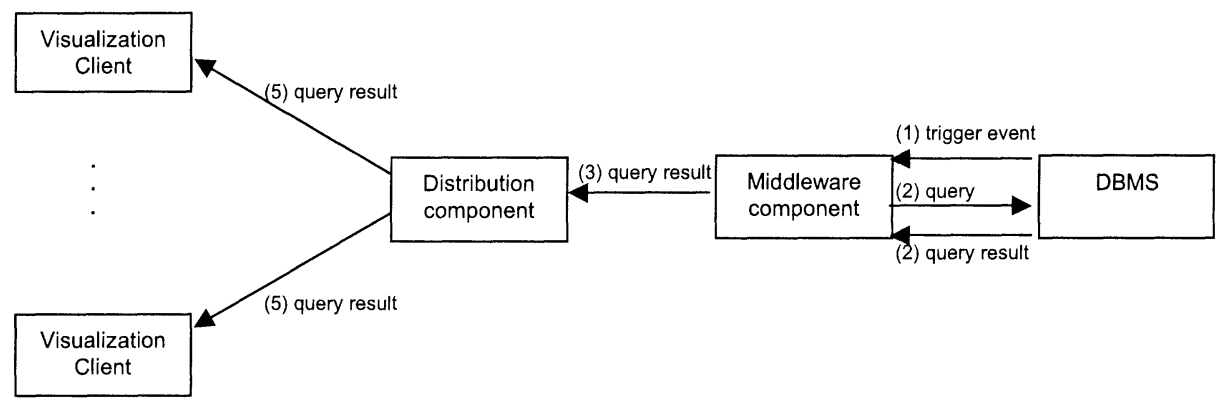

Figure 6: Architecture of a system in which a trigger causes a database query to be used to update data on several information visualization clients.

The last architecture described is a combination of the partial architectures depicted in Figure 3 and Figure 4. This architecture is shown in Figure 7. The action part of a trigger notifies the middleware component (1) which now checks if the triggering transaction has terminated regularly (2). If this is the case, the distribution component is notified (4). The distribution component then notifies the visualization clients (5). Only with this architecture a system in which actions are performed on the visualization clients in case a transaction has terminated regularly can be implemented.

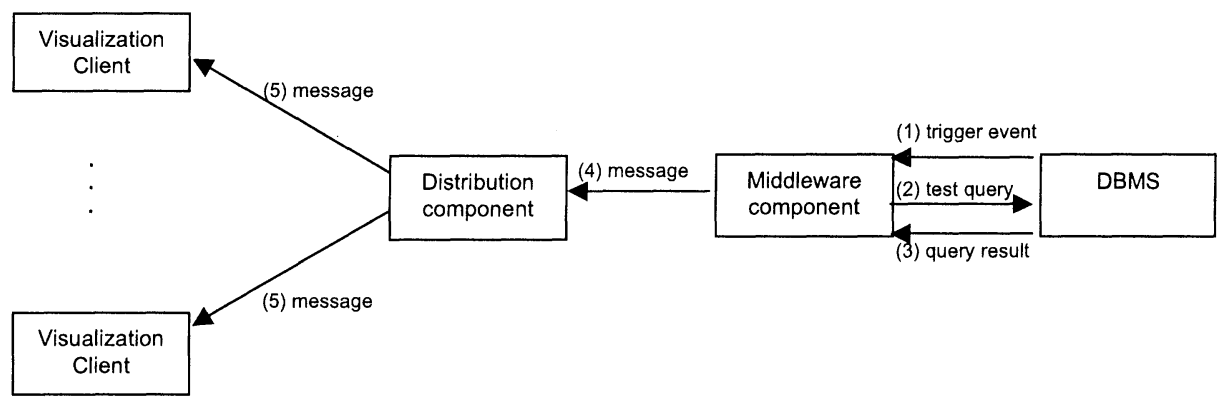

Figure 7: Architecture of a system in which the regular termination of the triggering transaction is tested. 


\section{A SYSTEM FOR THE NOTIFICATION OF VRML- WORLDS THROUGH DBMS TRIGGERS}

In this section, we describe a proof-of-concept prototypical implementation of a system for the notification of several VRML worlds running on client machines about modifications in a DBMS. The presented system is based on the observations in earlier sections and on the architecture in Figure 5 in which clients are notified about a possible change in the underlying database. The clients then read from the database and update their display based on the retrieved data.

The DBMS used in this specific implementation is the Informix Dynamic Server (IDS) which is a widely used relational database system. However, the implementation techniques used in the given scenario can be applied to most existing relational DBMS, available on the market today. The IDS implements the database trigger mechanism compliant to the SQL3 draft standard [ISO97b] and is therefore well suited for the given purpose.

Furthermore, we have used the standard web browser Microsoft Internet Explorer 4.01 and the VRML viewer control WorldView 2.1 for Internet Explorer from Intervista with the recommended JSAI patch installed as VRML-based information visualization clients. The operating system environment is Microsoft Windows NT 4.0 SP3. However, to assure a broad usability, no special browser features were used which exceed the VRML standard [ISO97a] and the EAI [Mar97].

\subsection{External Sensors}

If we want to notify a running VRML world through triggers there has to be a possibility to determine how the notification affects the VRML scene. We have chosen a solution which introduces a new VRML node type called ExternalSensor. This node provides VRML events through its EventOut fields when notified by a database trigger. However, this type of node can in fact be notified by any type of external system.

A ExternalSensor node in a VRML scene forms an interface to a socalled external sensor. An external sensor is an entity external to the VRML world which has a given name, an interface, and a behavior. The external sensors interface defines the data types for the values which can be sent to a VRML scene. An external sensor is typically a part of a running sensorserver process. A sensor server is a program to which VRML clients can log on and off.

The VRML syntax of the ExternalSensor node is as follows:

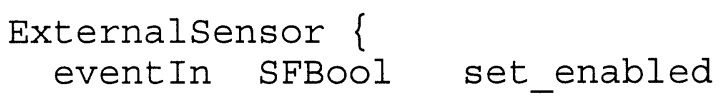




$\begin{array}{llll}\text { field } & \text { SFBool } & \text { enabled } & \text { TRUE } \\ \text { eventout } & \text { SFBool } & \text { enabled_changed } & \\ \text { field } & \text { SFString } & \text { sensorId } & " ~ \\ \text { field } & \text { SFString } & \text { server } & " 1 \\ \text { field } & \text { SFInt32 } & \text { port } & 22155 \\ \text { field } & \text { MFNode } & \text { eventouts } & \end{array}$

For the design of the ExternalSensor node the given sensor nodes from [ISO97a] were examined and the widely used ExposedField enabled was used. (In our case split up into an eventIn, a field, and a eventOut die to implementation issues). The eventIn set_enabled can be used for log on/off at a given external sensor. Sending TRUE (FALSE) logs on (off) to the external sensor. Within a log on the value TRUE is immediately available at the eventOut enabled_changed, which potentially occurs prior to the logon to the external sensor. Nodes which are connected to enabled_changed via ROUTE or nodes in the field EventOuts cannot be sure to receive all events from an external sensor after the receipt of TRUE from the event_changed field. That means notification messages can be lost in the beginning. As soon as FALSE is sent to set enabled, the transmission of events through the EventOuts field is immediately stopped, i.e., one does not wait until the log off process at the external sensor has finished.

Unlike the built-in VRML sensors, which have a clearly defined functionality, the node type ExternalSensor can be connected to different external sensors via the field sensorID. The value of the field sensorID is the unique name of an external sensor. Since external sensors are provided by sensor server it has to be determined on which server machine and on which port the server can be reached. This is achieved through the fields server and port where the value of server is the Internet address of the server and the value of port is the port number on which the sensor server is listening. The standard port number is 22155 which should not be occupied by other services (e.g., a web server) [Tan96].

The interface of an ExternalSensor node to match the interface of its external sensor is made available through the field EventOuts. This field contains nodes which in turn contain one single eventOut field. Therefore we have defined a node type named EventOutT for every existing basic VRML node type $T$ which contains an exposedField named value of the type $T$. As an example we give the syntax for the node type EventOutSFFloat:

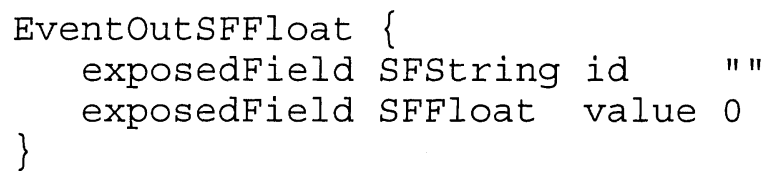


For each pair (N, T) from the interface of an external sensor a node with the type EventOut $\mathrm{T}$ with an id value of $\mathrm{N}$ can be inserted in the EventOuts field. An exposedField value provides an event if a message of interest is received from the external sensor. This is the case if a message contains a pair $(\mathrm{N}, \mathrm{W})$ in which $\mathrm{N}$ is the same string as in the nodes id field. If the interface of the external sensor is known, an appropriate node can be inserted to the interface in the field EventOuts. Given that sensor-id is the name of a sensor, server-address is the address of a sensor server, server-port is the port number, and $\left\{\left(\mathrm{N}_{1} \mathrm{~T}_{1}, \ldots, \mathrm{N}_{\mathrm{n}} \mathrm{T}_{\mathrm{n}}\right)\right\}$ is the interface of the sensor, then a node of the type ExternalSensor can be used as follows:

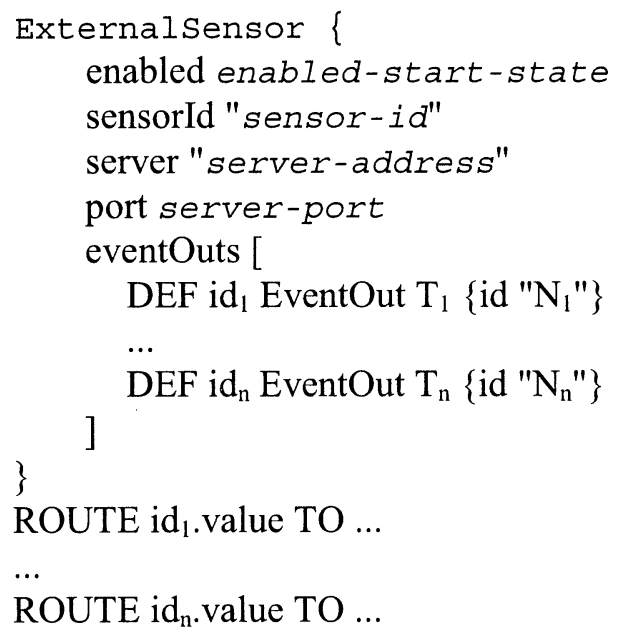

enabled-start-state determines if the node should be activated at the beginning. $i d_{1, \ldots}, i d_{n}$ are arbitrary identifiers. Note that not every possible pair of an external sensor interface has to have a corresponding node in the in EventOuts. It is enough to list the nodes which are really used in a VRML scene.

An example for the use of an ExternalSensor node is given below. Three temperatures from tree different locations $\mathrm{A}, \mathrm{B}$, and $\mathrm{C}$ are being visualized in a VRML scene. The sensor corresponding is named "temperature", the server is named "tempserver", and the port number is 22155. The exact visualization is not further detailed here but the temperatures are made available as three eventOut fields with the type SFFloat.

The interface of the external sensor is as follows:

\begin{tabular}{|c|c|c|}
\hline Sensor temperatur \\
\hline Name & Typ \\
\hline temperatur_A & SFFloat \\
\hline
\end{tabular}




\begin{tabular}{|c|c|}
\hline temperatur B & SFFloat \\
\hline temperatur C & SFFloat \\
\hline
\end{tabular}

The external sensor is used in the VRML scene as follows:

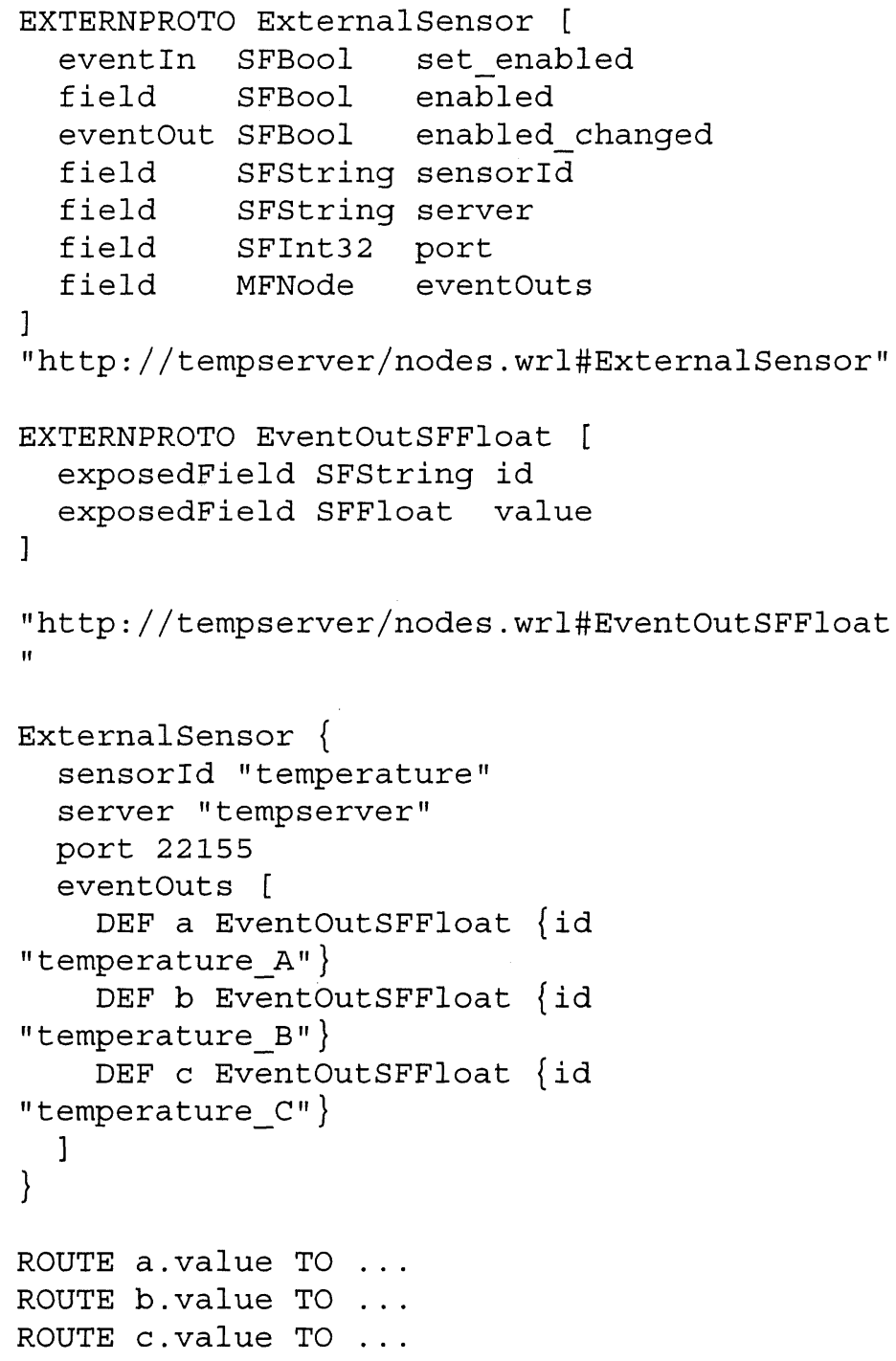

\subsection{Communication Protocol}

The communication between VRML nodes of the type ExternalSensor and an external system is handled by a protocol which is based on the well- 
known transmission control protocol (TCP). As soon as a VRML client wants to communicate with a sensor server, it establishes a TCP connection to the server and communicates with it via defined messages. If a VRML client wants to be notified about certain events (which can be different for every sensor) after registering with a sensor server, it has to keep the connection to the server open because the connection is used for the notification.

The messages are text-based and coded using ISO-8859-1 [ISO87]. Possible messages include requests for information about an external sensor, registering/unregistering with a sensor, sending data to a VRML client, and closing the connection. The exact syntax of the message format is not covered within the scope of this paper.

An example for communication messages based on this protocol are given below. It is based on the example in section 7.1:

Register with the temperature server for the external sensor temperature:

REGISTER temperature \#

Unregister with the temperature server for the external sensor temperature:

UNREGISTER temperature \#

Request the interface of the sensor temperature:

GETINFO temperature \#

Temperature server sends the interface of the external sensor temperature:

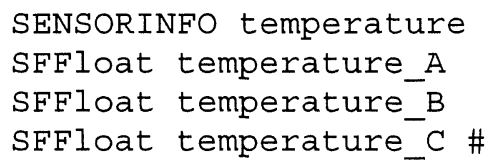

Temperature at location A has changed:

EVENT temperature

temperature_A 22.5 \#

All temperatures have changed:

EVENT temperature

temperature_A 22.5 
temperature_B 27.2

temperature_C 19.8 \#

\subsection{Trigger Server}

The trigger server we have implemented for the notification of VRML scenes through database triggers can be looked at as a special case of a sensor server. The trigger server is configured through a file which stores which trigger events are permitted from which machines. It is also possible to determine that a trigger event can come from any machine. With this feature, we can assure that no events can be faked and sent from machines other than the database server. The file format is very simple: A configuration file contains a list of names of trigger servers followed by an internet address or *. The character $*$ is a wildcard for all machines. An example for a configuration file in which two trigger sensors are defined follows:

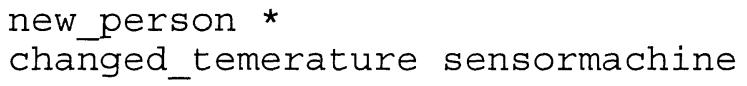

A new trigger sensor new_person is defined which accepts trigger events from an arbitrary machine are accepted. The new trigger sensor changed_temperature, however, only accepts messages from the machine with the address "sensormachine".

Note: If the VRML browser component only allows network connections to the machine from which the VRML scene was downloaded, as is the case with InterVista's WorldView 2.1, the trigger server must too run on this machine.

\subsection{Notification Procedure}

The procedure notify is defined and stored as part of an IDS database. This procedure is used to inform a trigger server within the action part of a database trigger in a system containing a database, a trigger server, and VRML clients. It has the following appearance:

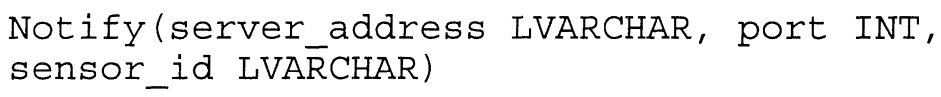

The procedure notifies a trigger server with the address trigger_server running on port port about the occurrence of an event within the sensor sensor_id. The trigger server is therefore instructed to inform all VRML clients which are registered for the sensor sensor_id. 


\subsection{Implementation Architecture}

The overall implementation architecture which uses the components described above to notify VRML worlds about changes in a database is displayed in Figure 8. The system contains VRML worlds, a trigger server, and a DBMS. The files containing the VRML world are requested from a web server which is not explicitly depicted in the figure because it does not participate in the notification process. The VRML worlds register with a trigger server to be notified by a certain trigger. A registration is confirmed by the trigger server to inform a VRML world of the exact point in time at which it surely begins to receive all trigger events from the database system. As a reaction, the VRML world can start a database query to read its data and start the visualization process. Thereafter the data can be read anew each time a trigger event occurs.

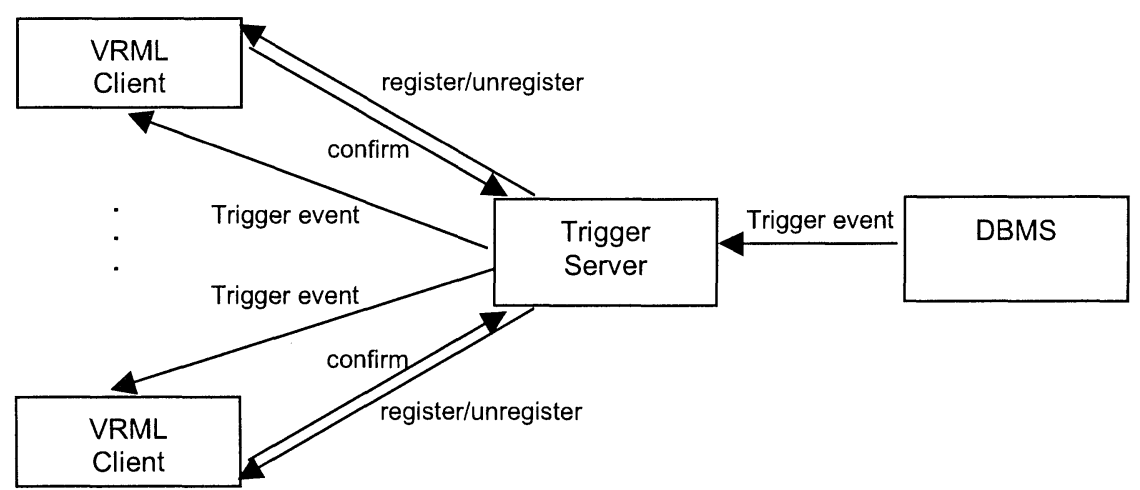

Figure 8: Implementation architecture which uses the described components to notify VRML worlds about changes in a database

If a VRML world does not want to receive any more trigger events, it can unregister with the trigger server. A VRML world can therefore register and unregister multiple times with a trigger server.

If the action of the trigger is executed, the trigger server is notified via the notify() procedure. The trigger server then notifies all VRML worlds registered for this event.

\section{CONCLUSIONS AND OUTLOOK}

We have outlined several possibilities of how a system could be structured in which database triggers are used to update information visualization client applications on changes of the underlying database. Furthermore, we have described components which have been designed and 
prototypically implemented. These components can be used in the development of a system in which VRML scenes are notified through database triggers. The use of these components should dramatically ease the development process in such a scenario. In particular, a server has been developed at which VRML clients can log on to be informed about possible changes in a database. A procedure which is stored in an Informix Dynamic Server DBMS has been developed. This procedure allows triggers stored in a database to notify the server. Furthermore, a VRML node type has been developed that can be used in arbitrary VRML scenes which are interested in database trigger notification. This node type performs all necessary actions to $\log$ on to the server component and is also responsible for the transmission of VRML events as a reaction to trigger notification. The new node type can even be used in a more general way. Notifications coming from arbitrary systems in the internet can be included into a VRML scene using this node. Therefore a protocol has been developed that handles the communication between these systems and the VRML scene. The reason for the universality of our solution for connecting VRML clients to external systems is that we wanted to additionally support potentially complex scenarios containing trigger usage or general active database systems for the update of VRML scenes.

In many areas more work has to be done. In the following some of these tasks are enumerated:

- Up to now the developed components have only been tested against very simple scenarios. The components clearly have to be tested in real world application environments to gain more detailed insight about their robustness and usability.

- Only a simple notification mechanism has been implemented: In a message no information about the modified data is transferred to the VRML clients. Furthermore, it is not assured that a modification has really taken place. A system as described in Section 6.4, in which an external component tests the database if the triggering transaction has terminated regularly, could be implemented.

- TCP based protocols have been used for the communication between the components. It still remains to be examined if there is another protocol which has clear advantages over TCP in this environment. Particularly multicasting could be used to inform the clients because all clients interested in a particular trigger notification recieve the same message upon trigger execution.

- It has to be analyzed how a system based on the developed components reacts to a failure of one or more system components. 
The results of such an analysis could directly flow into the further development of the components

- The general performance of systems based on the described components could be analyzed. For example, the time between the triggering database query and the actual screen update on the client could be measured. Furthermore it could be analyzed how many clients can be supported in parallel by the trigger server component. With these results the scope for the usage of the components could be determined more accurately.

\section{REFERENCES}

\section{BBKZ93}

Branding, H.; Buchmann, A.; Kudrass, T.; Zimmermann, J.: Rules in an Open System: The REACH Rule System. In Paton, N.; Williams, M. (Eds): Rules in Database Systems: Proceedings of the 1st International Workshop on Rules in Database Systems, Buc94

Edinburgh, Scotland, 30 August - 1 September 1993. Springer, 1994.

Buchmann, A.: Active Object Systems. In Dogac, A.; Öszu, M. T.; Biliris, A.; Sellis, T. Day95

(Eds): Advances in Object-Oriented Database Systems. Berlin, u. a.: Springer, 1994.

Dayal, U.: Ten Years of Activity in Active Database Systems: What Have We

Accomplished?. In: Berndtsson, M.; Hansson, J. (Eds): Active and Real-Time Database Systems (ARTDB-95): Proceedings of the First International Workshop on Active and

Real-Time Database Systems, Skövde, Sweden, 9-11 June 1995. London u. a.: Springer, 1996.

\section{DBC96}

Dayal, U.; Buchmann A.; Chakravarthy, S.: The HiPAC Project. In: Widom, J.; Ceri, S.

(Eds): Active Database Systems: Triggers and Rules For Advanced Database Processing.

\section{DGG95}

San Francisco: Morgan Kaufmann, 1996.

Dittrich, K.; Gatziu, S.; Geppert, A.: The Active Database Management System Manifesto: A Rulebase of ADBMS Features. In Sellis, T. (Ed): Rules in Database Systems: Second International Workshop, RIDS '95, Glyfada, Athens, Greece, EN94 September 25-27, 1995, Proceedings. Berlin u. a.: Springer, 1995.

Elmasri, R.; Navathe, S.: Fundamentals of Database Systems. Second Edition. Redwood GJ96

City, California u. a.: Benjamin/Cummings, 1994.

Gehani, N.; Jagadish, H.: Active Database Facilities in Ode. In: Widom, J.; Ceri, S.

(Eds): Active Database Systems: Triggers and Rules For Advanced Database Processing. HCD98

San Francisco: Morgan Kaufmann, 1996.

Hanson, E.; Chen, I.; Dastur, R.; Engel, K.; Ramaswamy, V.; Tan, W.; Xu, C.: A flexible and recoverable client/server database event notification system. Z. The VLDB Journal, ISO87 $7(1)$, February 1998, S. 12-24

ISO/IEC 8859-1:1987 Information technology -- 8-bit single-byte coded graphic character sets -- Part 1: Latin alphabet No. 1. 
ISO97a

ISO/IEC 14772-1:1998 Information technology -- Computer graphics and image processing -- The Virtual Reality Modeling Language -- Part 1: Functional specification ISO97b and UTF-8 encoding.

ISO-ANSI Working Draft: Database Language SQL -- Part 2: Foundation, October 1997. $\mathrm{X} 3 \mathrm{H} 2-97-315$.

\section{LHN99}

Leissler, M., Hemmje, M., Neuhold, E.: Supporting Image-Retrieval by Database Driven Interactive 3D Information-Visualization Proceedings of the VISUAL'99, Third Mar97 International Conference on Visual Information Systems, Amsterdam, June 1999

Marrin, C.: Proposal for a VRML 2.0 Informative Annex: External Authoring Interface Reference, November 21, 1997. http://www.vrml.org/ WorkingGroups/vrml-eai/proposals/chris/ExternalInterface.html.

\section{MLHN99}

Müller, A., Leissler, M., Hemmje, M., Neuhold, E.: Towards the Virtual Internet Gallery Proceedings of the IEEE Multimedia Systems '99 International Conference On Multimedia Computing And Systems, Florence, June 1999

\section{MSSNHPL98}

Massari, A., Saladini, L., Sisinni, F., Napolitano, W., Hemmje, M., Paradiso, A., Leissler, M.: Virtual Reality Systems For Browsing Multimedia In: Furth, B. (ed.): Handbook of Multimedia Computing 1998

\section{RLHA98}

Risse, T., Leissler, M. , Hemmje M., Aberer, K.: Supporting Dynamic Information Visualization With VRML and Databases. Proceedings of CIKM '98, Workshop on New Paradigms in Information Visualization and Manipulation, Bethesda, November 1998

\section{VDBWG97}

VRML Database Working Group: VRML Recommended Practices for SQL Database Access: Request for Proposals, August 22, 1997.

\section{WC96a} http://www.vrml.org/WorkingGroups/dbwork/dbrfp.html.

Ceri, S.; Widom, J.: Standards and Commercial Systems. In: Widom, J.; Ceri, S. (Eds): Active Database Systems: Triggers and Rules For Advanced Database Processing. San Francisco: Morgan Kaufmann, 1996.

\section{WC96b}

Widom, J.; Ceri, S. (Eds): Active Database Systems: Triggers and Rules For Advanced WC96c Database Processing. San Francisco: Morgan Kaufmann, 1996.

Widom, J.; Ceri, S.: Introduction to Active Database Systems. In: Widom, J.; Ceri, S. (Eds): Active Database Systems: Triggers and Rules For Advanced Database Processing. San Francisco: Morgan Kaufmann, 1996.

\section{BIOGRAPHIES}

Martin Leissler is scientific member of the DELITE digital libraries research division at GMD-IPSI in Darmstadt, Germany. He holds a Master Degree and is candidate for a $\mathrm{PhD}$ degree at Department of Computer Science of the Technical University of Darmstadt. His research interests include interactive virtual environments, information visualization, user interfaces, multimedia, multimedia databases, 3D Graphics. In 1996 he worked as a Systems 
Engineer at BITservice GmbH, Bad Homburg. From 1994-1997 he worked as a assistant research associate at GMD-IPSI in the department for Visual Interaction Tools (VISIT) and from 1997 to 1999 as a research associate at GMD-IPSI in the research division for Webapplications and Website-publishing (TOPAS). Since Nov. 1999 he works in the DELITE digital libraries research division. Martin Leissler is working in the context of R\&D related to VRML-, and Web-technology-based information and visualization systems, as e.g., the internet virtual gallery project (i-VG), an information system for the online exhibition and trade of art which is based on a database supported information catalogue environment enabling navigation on multimedia document collections.

Matthias Hemmje is division manager of the DELITE digital libraries research division at GMD-IPSI in Darmstadt, Germany. He holds a diploma and a PhD degree from Department of Computer Science of the Technical University of Darmstadt. His research interests include information retrieval, multimedia databases, agent based user interfaces, virtual environments, information visualization, multimedia, evaluation of interactive systems. From 1987-1991 he worked as a Systems Engineer, Dept. for Computer Aided Testing at Gebr. Hofmann KG, Darmstadt, from 1991-1999 he worked as a research associate at GMD-IPSI in the deptartment for Visual Interaction Tools (VISIT) and in the research division for Open Adaptive Information Management Systems (OASYS). Matthias Hemmje is working in the context of R\&D related to VRML-, MPEG-, and XML-based information retrieval and visualization systems, as e.g., the internet virtual gallery project (I-VG), the Congress Online (CO) congress information system which is based on a database supported Information Catalogue Environment enabling navigation on multimedia document collections. Matthias Hemmje is a member of the Visual Information Retrieval Interfaces (VIRI) working group founded by Bob Korfhage at the Univ. of Pittsburgh and he is a member of the European working group on Foundations of Advanced 3D Information Visualization (FADIVA).

Erich J. Neuhold received his M. S. in Electronics and his Ph.D. degree in Mathematics and Computer Science at the Technical University of Vienna, Austria, in 1963 and 1967, respectively.

From 1963 to 1972 he was a research scientist at the IBM Corporation in Vienna and the USA. There he was working on program languages, translators, formal description techniques and operating systems. From 1972 to 1983 he was Professor of Computer Science, Chair of Application Software, at the University of Stuttgart, Germany. His research field included distributed database and information systems, formal description systems for presentation of complex software products as well as software engineering.

In the years 1983 and 1984 he was Director of the Information Management Laboratory and later of the Systems Software Laboratory at Hewlett Packard, Palo Alto, USA. There he also worked in the areas of distributed operation systems and communication systems.

From 1984 to 1986 he was Professor of Computer Science, Technical University of Vienna, Institute for Applied Informatics and Systems Analysis. His work included distributed databases, knowledge-based systems and object-oriented approaches in these fields. 


\section{VISUAL DATABASE SYSTEMS}

In 1986 he was appointed Director of the newly founded Institute for Integrated Publication and Information Systems of the German National Research Center for Information Technology in Darmstadt, Germany. His primary research and development interests are in heterogeneous interoperable database systems, object-oriented multimedia knowledge bases and intelligent information retrieval. He also guides research and development in user interfaces including virtual reality concepts for information visualization, computer supported cooperative work, virtual meetings and conferences as well as integrated publication and information systems with special emphasis on multimedia hyperdocuments and on information mining in the Internet/WEB environment. National and international cooperation with research and industrial partners ensures the transfer of results into widely available prototypes and products.

Since 1989 he is also Professor of Computer Science, Integrated Publication and Information Systems, at the Darmstadt University of Technology, Germany.

Publications: 190 papers, 4 books, 9 edited books. 